\title{
SIMULTANEOUS PHOTOMETRY AND SPECTROSCOPY OF THE RAPID VARIABILITY OF THE BE STAR $\eta$ CEN *
}

\author{
S. ŠTEFL \\ Astronomical Institute, Academy of Sciences of the Czech Republic, \\ CZ-251 65 Ondřejov, Czech Republic \\ D. BAADE \\ European Southern Observatory \\ Karl-Schwarzschild-Str. 2, D-85748 Garching bei München, Germany \\ P. HARMANEC \\ Astronomical Institute, Academy of Sciences of the Czech Republic, \\ CZ-251 65 Ondřejov, Czech Republic \\ and \\ L. A. BALONA \\ South African Astronomical Observatory \\ P.O. Box 9, Observatory 7935, Cape Town, South Africa
}

\section{Introduction and observational results}

The reality of the previously reported triple-wave light curve (Cuypers et al. 1989, A\&A Suppl. 81, 151), and relations between rapid line profile and brightness variations of the B1-2III-Ve star $\eta$ Cen (HD 127972) were investigated by means of simultaneous high-resolution spectroscopy (4 nights, 86 Si III $455.2622 \mathrm{~nm}$ profiles) and two-station $u v b y$ photometry (14 nights) secured in 1992. To this database were added the uvby photometry by Cuypers et al. and unpublished $b$ observations by L.A.B. from 1988-1991.

After prewhitening for seasonal and medium cyclic $(\approx 30$ days $)$ changes, all $887 b$ data of 1987-1992 can be reconciled with a period of $0.642424 \mathrm{~d}$ according to the ephemeris:

$$
T_{\text {max.light }}=(\mathrm{HJD} 2448767.582 \pm 0.012)+\left(0^{d} .6424241 \pm 0^{d} .0000027\right) \times E
$$

The scatter is not reduced for either a double- or triple-wave period. There are systematic cycle-to-cycle variations of the light curve but no other coherent periodicity or "flickering" with an amplitude larger than about $0^{m} 005$. The full amplitude of rapid brightness variations is $\approx 0 r^{m} 10$ in $u$ and $0 r^{m} 06$ in $v, b$ and $y ; b-y$ varies only slightly while the $c 1$ index varies for $0 .^{m} 04$. The star is bluest in $u-b$ when brightest.

* Based on observations obtained at the European Southern Observatory, La Silla, Chile and the South African Astronomical Observatory 
The RV of the outer wings of the Si III line varies nearly sinusoidally with the $0.6424 \mathrm{~d}$ period and peak-to-peak amplitude of $62 \pm 5 \mathrm{~km} \mathrm{~s}^{-1}$. Similar periodicities can also be detected in $W_{\lambda}, 1^{\text {st }}$ and $3^{\text {rd }}$ moment, $I_{c}$, and FWHM. The object is brighter and bluer close to the RV maximum, when also $W_{\lambda}$, FWHM, and $I_{c}$ attain their maxima.

The Si III line profiles vary significantly on a time-scale of minutes. Moving bumps, carried across the line profile from blue to red, obviously cause the large part of the observed variability. They are barely visible near the line center where most of them disappear or weaken substantially. The appearance of the strong bumps apparently correlates with the phase of light minimum. Free fits of the composite velocity curves of individual travelling bumps led to periods of $0.683 \pm 0.017$ and $0.654 \pm 0.042$ days for the absorption and emission bumps, respectively, similar to the photometric period.

\section{Confrontation with current models}

Corotating surface or circumstellar structures in a single star can hardly be reconciled with periodic RV variations. Neither do they explain the observed profile modulation which is stronger in the wings than at the centre.

The nonradial pulsation (NRP) model explains the moving bumps by analogy to other early-type stars. It can also address the light and RV variations and make plausible the observed increased strength of the moving bumps in the line wings if the horizontal-to-vertical velocity amplitude ratio is large. The enormous difference between the amplitude of the $T_{\text {eff }}$ variations as determined from $u-b$ and $W_{\lambda}$ of Si III $455.3 \mathrm{~nm}(1200 \mathrm{~K}$ vs. $6000 \mathrm{~K})$ can be understood in terms of NRP if effects of varying microturbulence and electron pressure are included. If the pulsation period is of the order of several days in the corotating frame, the amplitude of $g$-modes cannot exceed a few $\mathrm{km} \mathrm{s}^{-1}$ in order to be consistent with the observed amplitudes of the photometric and spectroscopic variations. It is furthermore not clear that a pure NRP model can explain the large RV variations observed or the presence of higher-order bumps in just one half of the profile at a time.

Orbital motion in a binary system is the only straightforward interpretation for the sinusoidal RV curve observed. An orbital solution leads to a contact binary with Roche lobe radii of about $4 R_{\odot}$ and $1.3 R_{\odot}$, respectively. The model does not offer any simple explanation for the relative phasing of the RV and light curves observed and no direct spectroscopic evidence of the secondary was found. - The available data cannot discriminate between the pulsating-star and binary model which are based on almost disjunct sub-volumes of the observational parameter space.

(The full version of the paper will be submitted to Astron. 8 Astrophys.) 